\title{
The Effect of Foam Rolling for Three Consecutive Days on Muscular Efficiency and Range of Motion
}

\author{
Lewis J. Macgregor ${ }^{1 *}$ (D), Malcolm M. Fairweather ${ }^{2}$, Ryan M. Bennett ${ }^{1}$ and Angus M. Hunter ${ }^{1}$
}

\begin{abstract}
Background: Foam rolling (FR) has been shown to alleviate some symptoms of exercise-induced muscle damage and has been suggested to increase range of motion (ROM) without negatively impacting strength. However, it is unclear what neuromuscular effects, if any, mediate these changes.

Methods: In a randomized, crossover design, 16 healthy active males completed 2 min of rest or FR of the knee extensors on three consecutive days. Mechanical properties of vastus lateralis $(\mathrm{VL})$ and rectus femoris (RF) were assessed via Tensiomyography. Knee extension maximal voluntary contraction (MVC) and knee flexion ROM were also assessed, and surface electromyography amplitude (RMS) was recorded during a submaximal isometric contraction (50\% of MVC). Measures were performed before and after $(0,15$, and $30 \mathrm{~min}) \mathrm{FR}$ or rest.

Results: MVC was reduced on subsequent days in the rest condition compared to FR $\left(p=0.002, p \eta^{2}=0.04\right)$; $R O M$ was not different across time or condition $\left(p=0.193, p \eta^{2}=0.01\right)$. Stiffness characteristics of the VL were different on the third day of FR $\left(p=0.002, p \eta^{2}=0.03\right)$. RMS was statistically reduced 0,15 , and 30 min after FR compared to rest $\left(p=0.006, p \eta^{2}=0.03 ; p=0.003, p \eta^{2}=0.04 ; p=0.002, p \eta^{2}=0.04\right)$.

Conclusions: Following FR, MVC was elevated compared to rest and RMS was transiently reduced during a submaximal task. Excitation efficiency of the involved muscles may have been enhanced by FR, which protected against the decline in MVC which was observed with rest.
\end{abstract}

Keywords: Electromyography, Maximal voluntary contraction, Self-massage, Tensiomyography

\section{Key Points}

- FR reduced the muscle excitation required to complete a submaximal task, which led to reduced fatigue after 3 days. Muscle mechanical properties were altered following 3 days of FR.

- FR may be used for a sustained period to alter muscle mechanical properties and to enhance muscular efficiency.

- Participants were all healthy and active, so it is not clear if FR would have the same effect among clinical populations.

\footnotetext{
* Correspondence: I.j.macgregor1@stir.ac.uk

${ }^{1}$ Faculty of Health Sciences and Sport, University of Stirling, Stirling, UK Full list of author information is available at the end of the article
}

\section{Background}

In recent years, self-massage through foam rolling (FR) has become an increasingly popular treatment in managing muscular injuries and pain [1-3]. FR involves an individual applying their own body weight to a neoprene-coated cylinder, using small repetitive undulating movements to exert pressure on the muscle. Potential mechanisms of action for FR can be divided into two categories: first mechanical, focussed around alterations in the structure or state of fascial tissue; or second neurophysiological, focussing on afferent signaling from mechanoreceptors [4]. As the majority of FR research has focused on functional outcomes, or has involved FR subsequent to exercise-induced muscle damage, it remains difficult to ascertain which, if any, of these proposed mechanisms holds true.

Specific aims of FR have emerged around enhancing recovery and sporting performance $[5,6]$. Observed 
effects of FR have included reduced delayed-onset muscle soreness following physical activity $[7,8]$. It has also been reported that there are reduced decrements in muscle function subsequent to a bout of eccentric exercise, when treated with FR [9]. Also, FR has reduced knee extensor electromyography (EMG) amplitude during a dynamic lunge, which may be interpreted as improved movement efficiency due to lower excitation being required for a given task [10]. However, to date, there has been little research into the application of FR as a preventative as opposed to a recovery tool $[9,11]$. Moreover, there remains much conjecture around the overall efficacy of FR, with mixed findings regarding the acute effect on muscle function $[12,13]$ and range of motion (ROM) [14-16].

Evidence suggests that combining deep tissue massage with static stretching results in reduced spinal reflex excitability without altering twitch contractile properties during treatment, while stretching alone can prolong electromechanical delay, which remains unaffected by massage [17]. Therefore, whilst stretching increases ROM through both neural and mechanical factors, massage-induced alterations can more likely be attributed to reflex inhibition [17]. However, in our laboratories, we have linked massage to muscle force impairment alongside maintained neuromuscular recruitment [18]; as a result of this, we proposed that observed force impairment was due to changes in muscle architecture and associated alterations in series compliance. While these architectural changes were not measured in the aforementioned study [18], such alterations would likely affect muscle mechanical properties [19], which can be measured from the extent of muscle radial displacement, through tensiomyography (TMG) [20]. To date, only two studies [21,22] have measured muscle mechanical properties, using TMG, on rested muscle, following FR, both studies reporting no acute change in muscle displacement, with measurements performed following a single session of FR. Given that anecdotal evidence suggests FR is typically used by athletes habitually performing prolonged and high-intensity exercise to break up trigger point adhesions and alleviate perceived muscle stiffness, it is important to asses if FR can attenuate exercise-induced changes in contractile properties, following repeated applications. However, to our knowledge, no study has examined the effect of more prolonged application of FR following exercise, across consecutive days, on muscle contractile properties.

If FR is to be effectively prescribed to treat muscle injuries and pain [5, 23-25], it is necessary to understand the mechanisms of action, in order to establish best practice. Therefore, the aim of the present study was to investigate muscle mechanical properties, strength and ROM over the course of three consecutive days of treatment with well-controlled bouts of FR. It was anticipated that the repeated demands of the testing procedure should induce detectable residual fatigue, as may be expected with day-to-day submaximal exercise. However, increased neuromuscular efficiency through FR would limit the symptoms of fatigue. Therefore, we hypothesized that FR would reduce muscle stiffness characteristics and increase ROM, without additional force decrement costs, in spite of fatigue-associated impairments in muscle function.

\section{Methods \\ Participants}

Based on a priori power analysis (G*Power 3 ) of predicted changes in ROM following self-massage, with $\alpha=0.05$ and $1-\beta=0.80$, sixteen healthy, recreationally active, male participants with no history of neuromuscular or musculoskeletal disorders were recruited to complete this study (age $24.5 \pm$ 4.4 years, height $1.8 \pm 0.1 \mathrm{~m}$, body mass $82.4 \pm 8.0 \mathrm{~kg}$, and knee extension strength (MVC) at baseline $222.0 \pm$ $36.5 \mathrm{~N} \mathrm{~m}$ ). Participants were not currently undertaking any form of self-massage at the time of their participation in the study and were provided with no prior indication as to the hypothesis regarding the potential effects of self-massage. Participants refrained from (1) any unaccustomed physical activity for the duration of the trial and (2) any strenuous exertion for at least $24 \mathrm{~h}$ prior to each testing session. Volunteers provided written consent, having been informed of any potential risks involved in their participation. The study was performed in accordance with the standards set by the latest revision of the Declaration of Helsinki and was approved by the local Research Ethics Committee.

\section{Study Design}

Following full familiarization of the testing procedures (utilizing the non-dominant leg), participants reported to the laboratory for two separate trials in a randomized, counterbalanced crossover design to incorporate two different interventions; one intervention was foam rolling while the other was in the rested condition. Participants were assigned, via block randomization, to decide the order in which trials were completed. Each trial consisted of three testing sessions on three consecutive days, with 7 days separating the start of each trial. Participants reported to the laboratory following an overnight fast and initially rested in a supine position for $30 \mathrm{~min}$. Following this rest period, mechanical and contractile properties of the vastus lateralis $(\mathrm{VL})$ and rectus femoris $(\mathrm{RF})$ were measured using tensiomyography (TMG: BMC Ltd., Ljubljana, Slovenia). Participants were then tested for knee flexion range of motion (ROM) before isometric strength assessments, using an isokinetic dynamometer (Kin Com, Chattanooga, Hixson, TN, USA). Following baseline measures, participants either rested for $2 \mathrm{~min}$ or performed $2 \mathrm{~min}$ of self-massage (FR). All 


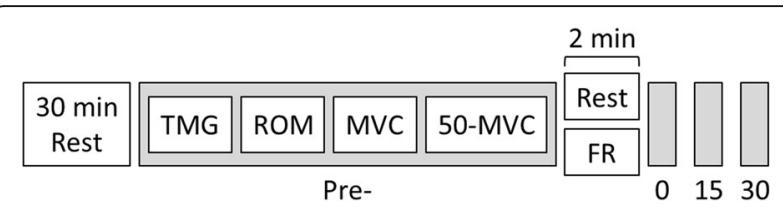

Fig. 1 Timeline of the experimental design. TMG = tensiomyography; $\mathrm{ROM}=$ range of motion; $\mathrm{MVC}=$ maximal voluntary contraction; $50-M V C=50 \%$ of maximal voluntary contraction; $F R=$ foam rolling

measurements were then repeated, in an identical order to pre-intervention: immediately, 15-min and 30-min post rest/FR (Fig. 1). All measurements were performed on the dominant leg. Dietary intake records were completed on the day preceding each session of the first trial, and participants were instructed to replicate their dietary intake before each visit for the second trial.

\section{Protocol}

After resting in a supine position for 30 -min participants then adopted a knee joint angle of $60^{\circ}\left(0^{\circ}=\right.$ full extension), which was maintained by the use of a foam support placed beneath the popliteal fossa. Two pairs of self-adhesive electrodes $\left(5 \mathrm{~cm}^{2}\right)$ (Axelgaard, USA) were affixed to the skin: one pair over the VL and one pair

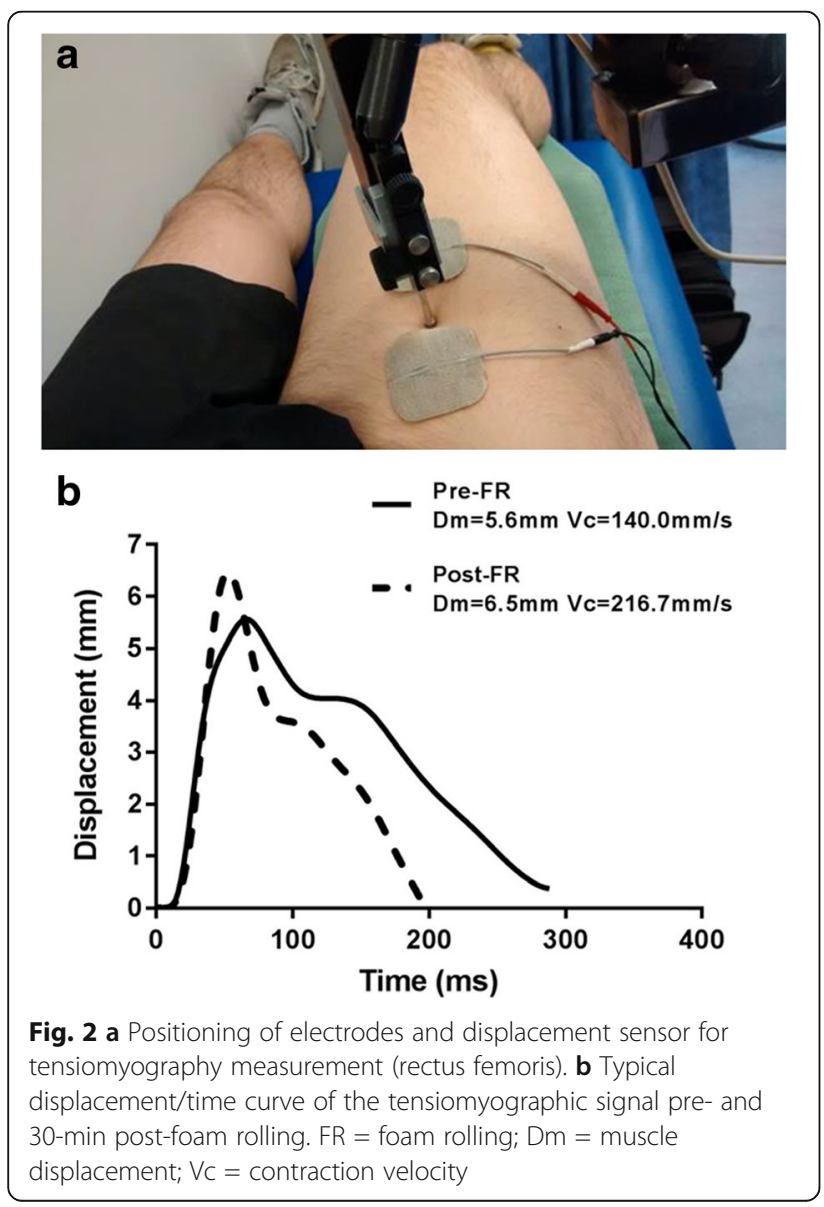

over the RF (Fig. 2a). A digital TMG displacement sensor (GK 40, Panoptik d.o.o., Ljubljana, Slovenia) was then positioned perpendicular to the muscle belly, equidistant between the pair of electrodes [26]. The sites over each muscle, of the sensor and the electrode pair, were marked with semi-permanent ink to enable exact relocation following FR treatment and on subsequent days $[27,28]$. A single 1-ms wide stimulation pulse was delivered, which applied initial current amplitude of $20 \mathrm{~mA}$. This amplitude was progressively increased by $10 \mathrm{~mA}$ increments until peak twitch response was obtained [26]. In order to minimize the effects of fatigue and potentiation, rest periods of $10 \mathrm{~s}$ were allowed between each stimulation pulse. Typical peak responses were observed at amplitude between 40 and $70 \mathrm{~mA}$, and only the output data for that particular stimulation intensity were used for analysis. Output parameters were extracted and analyzed from each peak twitch response [27]: Displacement (Dm), the extent of maximal radial deformation $(\mathrm{mm})$ of the muscle belly during contraction; Contraction velocity $(\mathrm{Vc})$, the rate $(\mathrm{mm} / \mathrm{s})$ of contraction between 10 and $90 \%$ of maximal displacement $[\mathrm{Vc}=\mathrm{Dm} 80 / \mathrm{Tc}]$ where $\mathrm{Tc}=$ contraction time between 10 and $90 \%$ of peak radial displacement of the muscle belly; Dm80 = the radial displacement occurring during the time period of Tc [26] (Fig. 2b).

Knee flexion ROM was measured in accordance with previous literature [9]. Participants adopted a modified kneeling lunge position. The non-dominant leg was positioned with the sole of the foot flat on the floor and the knee flexed to $90^{\circ}$; participants were permitted to place their hands on this knee for support, but were instructed to angle their torso perpendicular to the floor throughout the ROM assessment. With the hip of the dominant leg extended as far as possible, the foam roller was placed under the ankle in order to standardize the starting position. Internal knee angle was recorded, using a goniometer, and then, the knee was flexed as far as voluntarily possible. Maximal knee flexion was held only as long as was required to measure the internal angle for a second time. Total ROM was taken as the difference between starting knee angle and end knee angle. Intraclass correlation coefficient (ICC) between baseline ROM at the start of each trial was 0.85 .

Participants were coupled to the isokinetic dynamometer and secured using nylon straps, according to the manufacturer's guidelines. Gravitational corrections were performed, in accordance with existing recommendations [29]. A pair of $\mathrm{Ag} / \mathrm{AgCl}$ self-adhesive electrodes (PNS Dual Element Electrode; Vermed, VT, USA) were affixed to the skin over the $\mathrm{VL}, 1 / 3$ of the distance from the greater trochanter to the lateral femoral epicondyle, with an inter-electrode distance of $20 \mathrm{~mm}$, following thorough preparation of the skin in accordance with 
SENIAM guidelines [30]. A reference electrode was affixed to the patella. These electrodes remained in place throughout the entire duration of the testing session. Surface EMG (sEMG) was recorded during all isometric contractions and was synchronized with torque output. sEMG was captured at $2 \mathrm{KHz}$, anti-aliased with an upper bandwidth of $500 \mathrm{~Hz}$ and band pass filtered automatically at $10-500 \mathrm{~Hz}$ using a fourth-order zero-lag Butterworth filter.

Participants performed a standardized warm up [31, 32] prior to baseline measurements. Maximal isometric voluntary contraction (MVC) was measured at a knee joint angle of $60^{\circ}\left(0^{\circ}=\right.$ full extension $)$; the limb was secured by a Velcro strap proximal to the medial malleolus. The angle of $60^{\circ}$ was chosen, as it lies within the well-established range of reported optimal knee joint angles, for peak isometric torque production [33]. Participants were instructed to exert peak force as quickly as possible and to hold each contraction for $5 \mathrm{~s}$. Consistent verbal encouragement was provided by the same investigator throughout, to ensure maximum effort. To maintain internal validity, the same two investigators were present throughout all testing [34]. Participants performed three contractions with 60-s recovery between each. The highest force output achieved was designated MVC and stored for analysis. ICC between baseline MVC at the start of each trial was 0.70. Participants next performed a 30-s submaximal isometric contraction at $50 \%$ of their baseline MVC (50-MVC). Torque was to be increased gradually until the target output was reached then held as steady as possible for $30 \mathrm{~s}$ (mean torque \pm SD: $104.2 \pm 10.7 \mathrm{~N} \mathrm{~m}$ and $102.7 \pm 10.1 \mathrm{~N} \mathrm{~m}$, during rest and FR trials respectively). Participants were provided with visual and verbal feedback throughout 50-MVC. This protocol was expected to result in moderate, but detectable symptoms of residual muscle fatigue, across the duration of the testing period. sEMG was captured for $30 \mathrm{~s}$ during 50-MVC; signals were RMS converted using the data collection software (Acknowledge ${ }^{\circ}$ 3.9.1, Biopac Systems Inc., Goleta, CA, USA) and normalized to sEMG RMS from the MVC. Normalized RMS were divided into $5 \times 6 \mathrm{~s}$ epochs for analysis.

\section{Foam Rolling}

Following baseline measurements, participants performed self-massage, using a commercially available foam roller (TriggerPoint Performance, Austin, TX, USA) constructed of a hollow PVC pipe surrounded by a thin layer of neoprene [35]. The foam roller was positioned initially at the mid-point between the anterior inferior iliac spine (AIIS) and the upper border of the patella; participants supported themselves upon their forearms. To ensure that there were no other ground contact points, participants were instructed to plantar flex, whilst also positioning their feet

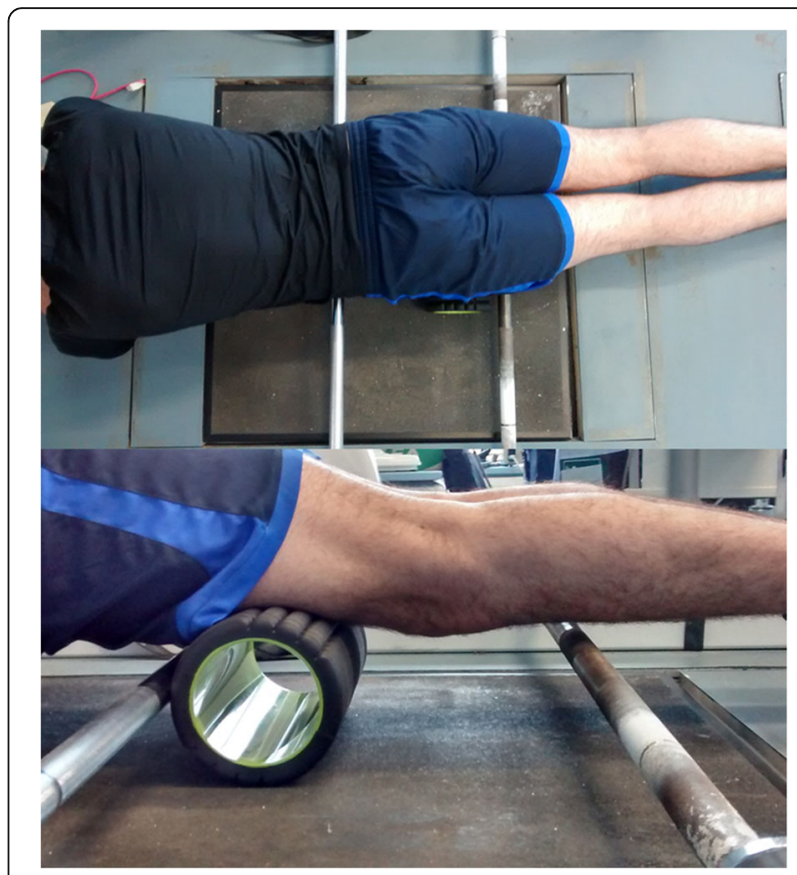

Fig. 3 A participant performing self-massage using the foam roller

and knees together, in order to focus the pressure of the foam roller upon the anterolateral aspect of the thigh (Fig. 3). The length of area that was treated with FR was $2 / 3$ of the distance between the AIIS and the upper border of the patella. A custom-built metal frame positioned beneath the participant ensured that the correct area was treated. Once in position, participants rolled backwards and forwards in an undulating motion; the rate of movement was controlled by a metronome set at a predetermined rate based on one complete roll of the treated area (proximal-to-distal or distal-to-proximal) per $1 \mathrm{~s}$. The foam roller was exclusively in contact with a force platform (400S Force Plate, Innervations, Australia), and force was recorded throughout the 2-min FR treatment using Acqknowledge software at a sampling frequency of $2 \mathrm{KHz}$ (Table 1). It has been suggested that stretching- and massage-based interventions may elicit a whole-body systemic response, such that treating one limb may nullify the effectiveness of the contralateral limb as a comparison [21, 24, 36]. Therefore, a separate (rest) condition was completed; however, as the timeframe of any residual effect of FR has not been established, the start

Table 1 Force applied during FR treatment

\begin{tabular}{lllll}
\hline & D0 & D24 & D48 & Overall \\
\hline Average force & $48.2 \pm 54.4$ & $45.3 \pm 50.6$ & $48.0 \pm 81.6$ & $49.3 \pm 62.9$ \\
rolled (N) & $(1.13)$ & $(1.12)$ & $(1.70)$ & $(1.28)$ \\
Average relative & $7.0 \pm 1.3$ & $6.6 \pm 1.0$ & $6.0 \pm 0.9$ & $6.5 \pm 1.1$ \\
force rolled (N/Kg) & $(0.19)$ & $(0.15)$ & $(0.15)$ & $(0.17)$ \\
\hline
\end{tabular}

FR foam rolling

Values are mean \pm SD (Coefficient of Variation \%), $n=16$ 
of the rest condition and FR trials were seperated by 7 days. During the rest condition, participants adopted a supine position with the popliteal fossa of their dominant leg resting upon the foam roller in order to maintain a knee joint angle of $\sim 60^{\circ}\left(0^{\circ}=\right.$ full extension $)$. The duration of the rest period was identical to the duration of FR.

\section{Statistical Analysis}

All data were tested for assumption of homoscedasticity (Levene's test) and normality (Ryan-Joiner test); residuals were assessed for linearity and normal distribution. Comparisons were performed between normalized RMS values (as described above); all other comparisons were performed using un-normalized data, analyzed using a three factor repeated measures (group [2] $\times$ time [4] $\times$ day [3]) analysis of variance (ANOVA) for normalized data; when analyzing un-normalized data, baseline values were included as covariates (ANCOVA). Tukey post hoc analysis was performed where appropriate (Minitab 16 statistical software, Minitab Ltd., Coventry, UK). Statistical significance was accepted at $p<0.05$. All values were reported as mean \pm standard deviation (SD), and partial $\eta^{2}$ effect sizes $\left({ }_{\mathrm{p}} \eta^{2}\right)$ were calculated by $\eta_{\mathrm{p}} \eta^{2}=\mathrm{SS}_{\text {conditions }} /\left(\mathrm{SS}_{\text {conditions }}+\mathrm{SS}_{\text {error }}\right)$.

\section{Results}

\section{Maximal Isometric Voluntary Contraction and Range} of Motion

A statistically significant interaction effect $\left(F_{(2,15)}=6.53\right.$, $p=0.002,{ }_{\mathrm{p}} \eta^{2}=0.04$ ) was detected in MVC across days and condition (Fig. 4); this was caused by the rest condition statistically declining over the full-time period whereas the FR was maintained $(p=0.024)$. ROM was not statistically different between conditions or over time $\left(F_{(3,15)}=1.58, p=0.193,{ }_{\mathrm{p}} \eta^{2}=0.01\right)$.

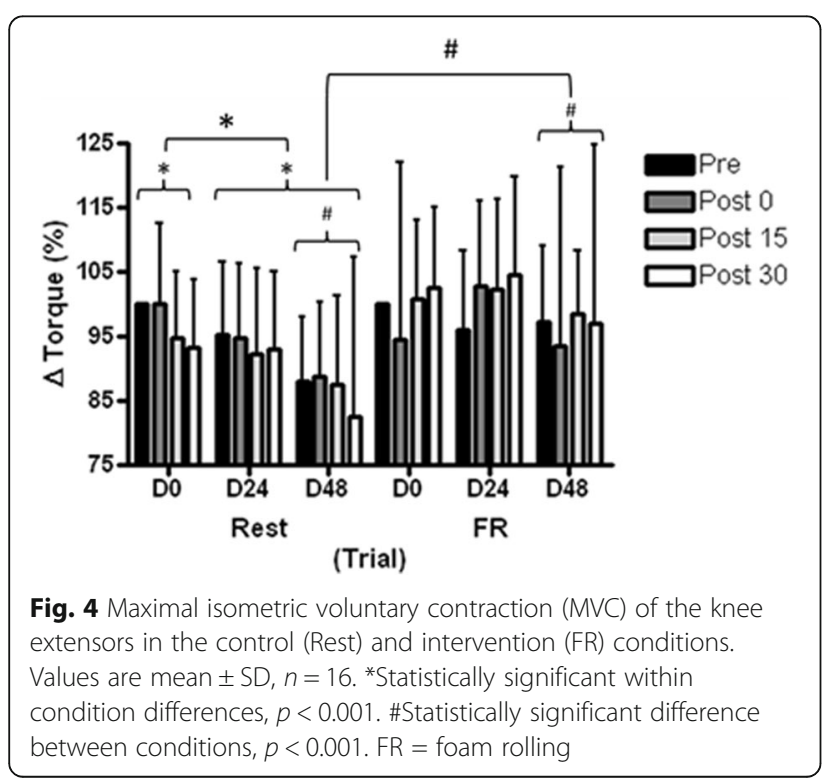

\section{Neuromuscular Recruitment}

On D24 and D48, FR statistically reduced RMS required for 30-s submaximal contraction, compared to rest. This reduction was observed during $0-6 \mathrm{~s}\left(F_{(2,15)}=12.62, p<0.001\right.$, $\left.{ }_{\mathrm{p}} \eta^{2}=0.07\right), 12-18 \mathrm{~s}\left(F_{(2,15)}=20.68, p<0.001,{ }_{\mathrm{p}} \eta^{2}=0.1\right)$ and $24-30 \mathrm{~s}\left(F_{(2,15)}=24.37, p<0.001, \mathrm{p} \eta^{2}=0.1\right)$, with statistically significant interaction effects $\left(F_{(3,15)}=4.18, p=0.006\right.$, ${ }_{\mathrm{p}} \eta^{2}=0.03 ; F_{(3,15)}=4.71, p=0.003,{ }_{\mathrm{p}} \eta^{2}=0.04 ; F_{(3,15)}=5.21$, $\left.p=0.002,{ }_{\mathrm{p}} \eta^{2}=0.04\right)$ respectively between time and condition. These were caused by statistically reduced RMS immediately, 15- and 30-min post-FR compared to post-rest $(p=0.031 ; p=0.049 ; p=0.039)$. Figure 5 shows the change in RMS between pre and 30 min post on each trial day in both rest and FR.

\section{Contractile Properties}

A statistically significant interaction effect was detected in peak displacement $(\mathrm{Dm})\left(F_{(2,15)}=6.32, p=0.002,{ }_{\mathrm{p}} \eta^{2}=0.03\right)$ and velocity $(\mathrm{Vc})\left(F_{(2,15)}=4.87, p=0.008, \mathrm{p} \eta^{2}=0.02\right)$ of VL with day and condition (Fig. 6), caused by statistically greater $\operatorname{Dm}(p=0.021)$ and $\mathrm{Vc}_{\mathrm{c}}(p=0.023)$ on D48 in the FR treatment condition compared to the rest condition. Dm $\left(F_{(2,15)}=2.17, p=0.116,{ }_{\mathrm{p}} \eta^{2}=0.01\right)$ and $\mathrm{Vc}\left(F_{(2,15)}=1.61\right.$, $\left.p=0.202,{ }_{\mathrm{p}} \eta^{2}=0.009\right)$ of RF were not statistically different between conditions or over time.

\section{Discussion}

This study demonstrated reduced EMG RMS, during sustained submaximal contraction, following FR, when compared to increased RMS following rest. MVC was maintained following FR, avoiding decreases observed throughout the rest condition. Contractile characteristics of RF were unaffected, as shown by unaltered TMG parameters, although reduced muscle stiffness characteristics, and increased contraction velocity were evident in VL following the third consecutive day of FR. It had been hypothesized that elevated Dm would be indicative of altered muscle architecture and thus be associated with augmented ROM; surprisingly however, ROM remained unchanged over time and did not differ between conditions.

Perhaps the greatest insight into the mechanisms behind the effects of FR has been provided by investigating neuromuscular recruitment during static fatiguing contractions; RMS was lower at all time points following FR compared to the same time points following rest. Within the rest condition, RMS increased from baseline up to 15 and $30 \mathrm{~min}$ post-rest, potentially indicating that the muscle required greater neural drive in order to maintain $50 \%$ of MVC [37], suggesting that submaximal fatigue occurred [38]. However, similar changes in RMS arise due to peripheral factors, such as metabolite accumulation [39]. Increased neural drive is indicated by increased RMS which occurs as higher threshold motor 

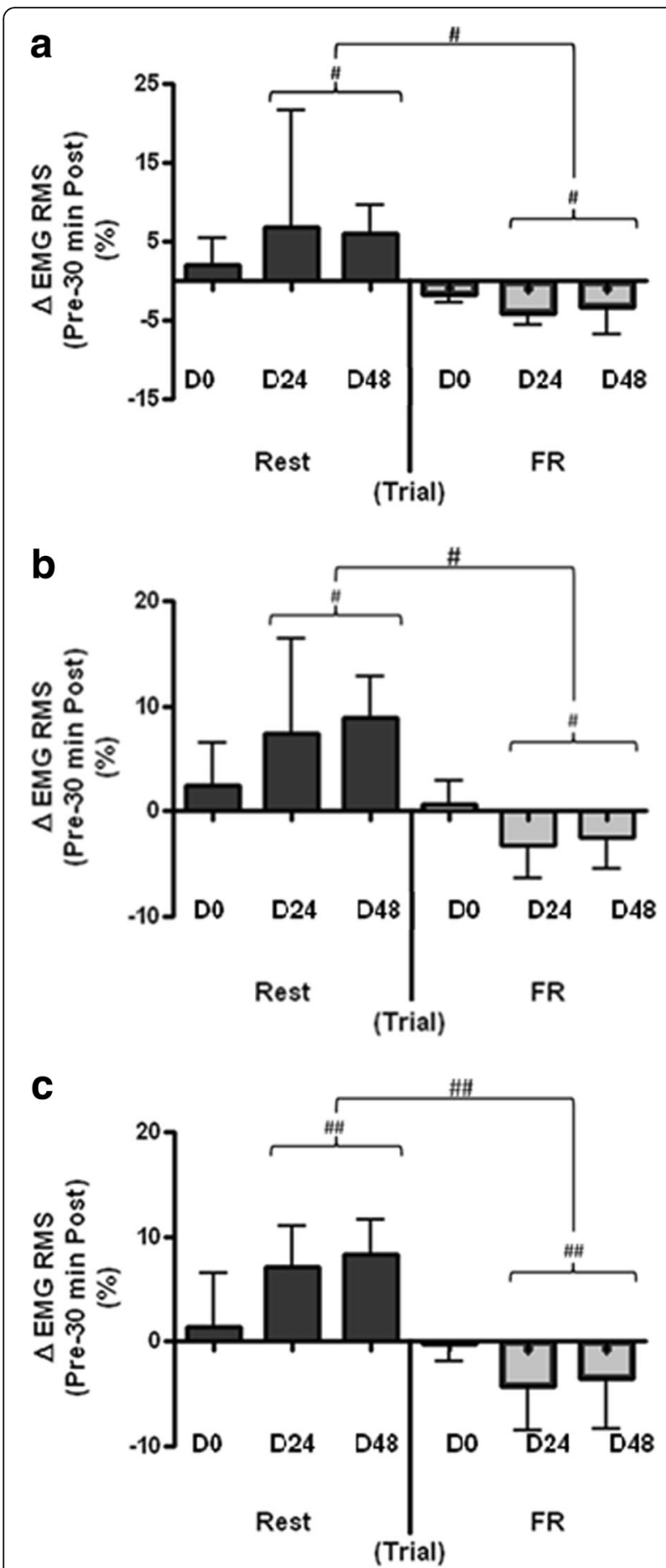

Fig. 5 Change in normalized RMS (a 0-6, b 12-18, and c 24-30 s) from pre- to 30-min post-treatment in the control (Rest) and intervention (FR) conditions. Values are mean $\pm \mathrm{SD}, n=16$ \#Statistically significant difference between conditions, $p<0.01$; \#\#Statistically significant difference between conditions, $p<0.001$. FR = foam rolling; EMG RMS = electromyography root mean squared

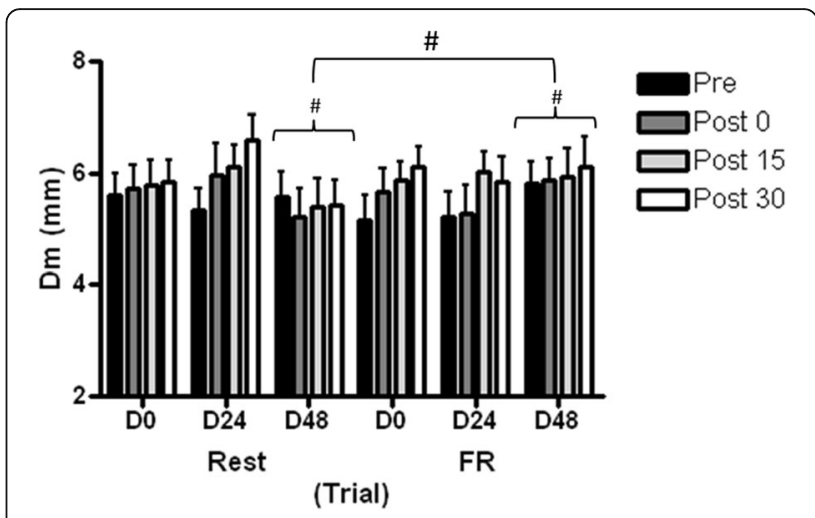

Fig. 6 Maximal radial displacement (Dm) of vastus lateralis $(V L)$ muscle belly. Values are mean $\pm \mathrm{SD}, n=16$. \#Statistically significant difference between conditions, $p<0.001$. $F R=$ foam rolling;

$\mathrm{Dm}=$ muscle displacement

units (MU) are recruited to sustain force production, in compensation for earlier recruited MUs becoming fatigued [40, 41]. In agreement with our hypothesis, these findings suggest that FR was able to reduce the impact of fatigue during this submaximal task, allowing the muscle to be activated more efficiently. Furthermore, on D48, RMS of the rest condition was higher than the previous 2 days, in addition to being elevated vs. FR condition, indicating there may have been chronic adaptation to repeated bouts of FR on consecutive days. However, given the constraints discussed above, it cannot be confirmed that changes in RMS were the result of altered neural drive, as opposed to peripheral factors. Future research should therefore incorporate $\mathrm{M}$-wave amplitude characteristics, and normalize EMG to M-wave, in order to neutralize the effect of peripheral muscle fatigue, and isolate factors relating to neuromuscular efficiency [39, 42, 43].

It seems plausible that sustained muscle lengthening under pressure, applied during FR, will stimulate muscle spindles and Golgi tendon organ [10, 17]. Stimulation of these mechanoreceptors would lead to increased activity of type Ib afferents, thereby leading to greater proprioceptive feedback from the muscle in question to the CNS, as has been demonstrated through altering muscle-spindle length following proprioceptive neuromuscular facilitation (PNF) $[44,45]$. The viscoelastic nature of skeletal muscle also may impact responses to FR [46, 47]. Indeed, shear wave velocity of knee flexors has been shown to decrease following FR; while this was not associated with increased $\mathrm{ROM}$, the combination of FR with active warm up prolonged observed increases in ROM beyond active warm up alone [48].

Peak torque (MVC) was elevated $30 \mathrm{~min}$ after FR, which was also higher than $30 \mathrm{~min}$ post-rest. The decline in MVC on D24 and D48 of the rest condition can be interpreted as another marker of the fatiguing 
effect of the test protocol. It follows that lower neural drive required to complete the submaximal task following FR, compared to rest, indicates less demand on the muscle and, as such, has enabled maintenance of MVC following consecutive days of FR. Previous studies have reported no alteration in strength or performance $[6,49]$ or improved performance through combining FR with a dynamic warm up compared to a dynamic warm up alone [13]. The present study is the first to report an improvement in MVC following FR alone. Previous studies have measured strength or performance $\leq 10 \mathrm{~min}$ after $\mathrm{FR}$; in accordance, we found no change in MVC immediately and $15 \mathrm{~min}$ post-FR. When force output is important, these findings suggest that for optimal effect, FR should be performed $\geq 30$ min prior to activity.

As FR has previously been reported to increase ROM without decrements in force production, one of the main aims of this study was to examine the contractile mechanics of muscles subjected to FR. It was hypothesized that muscle displacement would increase following consecutive days of FR, indicative of reduced muscle stiffness. Despite the lack of improvement in ROM, higher displacement was observed in VL on D48 of FR treatment compared to D48 of the rest condition. Furthermore, Dm was $15.7 \%$ greater than baseline, $15 \mathrm{~min}$ post-FR, and $19.0 \%$ greater than baseline 30 min post-FR on D48; compared to $3.7 \%$ lower and $2.8 \%$ lower at 15 and $30 \mathrm{~min}$ post-rest. Although no differences were revealed in RF, there was a tendency towards greater displacement following FR. Similarly, Murray et al. [21] reported no change in ROM; however, they also reported no effect of FR on TMG Dm, although only a single bout of FR was performed. Given that we observed a difference in Dm between groups only on D48, it may be speculated that continuing with FR treatment for a more extended period of time may lead to more pronounced effects on contractile properties. It should also be noted however, that in the present study, independent of day, VL displacement was greater 15 and 30 min post treatment, in both the rest and FR conditions. Therefore, it seems that the test protocol itself may have impacted upon muscle mechanical properties; in order to fully elucidate the impact of FR on mechanical properties, investigation of the impact of FR on muscle contractile properties in isolation from other measurements may be required, to avoid interference from additional stretch and fatigue. Furthermore, other researchers have described mixed results following FR protocols lasting between 1 and 3 weeks [50, 51]; a dose response may exist, such that prolonged FR treatment, lasting $\geq 1$ week, may prove more effective than acute FR, or treatments lasting $\leq 6$ days. Interestingly, shorter treatment periods, combining FR with complimentary techniques, such as static stretching [51] or active warm up [48] may augment the response. As such, future research should aim to explore the optimal combination of techniques to elicit neuromuscular outcomes.

\section{Conclusions}

This is the first study to illustrate elevated strength 30 min after a 2-min bout of FR, alongside reduced RMS during submaximal activity following FR. This reduced RMS protected the muscles from the fatiguing effects of the protocol, observed through the rest condition. Muscle displacement was increased after three consecutive days of FR; however, this did not translate into improved ROM. It seems that a single bout of FR may lead to alterations in neural drive. Such alterations potentially enhance strength and performance and delay the onset of fatigue.

\section{Abbreviations \\ Dm: Displacement; FR: Foam rolling; MVC: Maximal voluntary contraction; $\mathrm{p} \eta^{2}$ : Partial eta squared; RF : Rectus femoris; RMS: Root mean squared; ROM: Range of motion; sEMG: Surface electromyography; \\ TMG: Tensiomyography; Vc: Contraction velocity; VL: Vastus lateralis}

\section{Acknowledgements}

This research was funded by SportScotland: Scottish Institute of Sport. The authors acknowledge Mr. Chris Grigson for technical support and Ms. Helen Thomson for assistance during data collection.

\section{Funding}

This research was funded by SportScotland: Scottish Institute of Sport.

\section{Authors' Contributions}

LJM contributed to the study design, data collection, analysis, and writing of the text. MMF contributed to the writing of the text. RMB contributed to the data collection, analysis, and writing of the text. AMH contributed to study design, analysis, and writing of the text. All authors read and approved the final manuscript.

Ethics Approval and Consent to Participate

The study was performed in accordance with the standards set by the latest revision of the Declaration of Helsinki and was approved by the University of Stirling Sports Studies Ethics Committee. Volunteers provided written consent, having been informed of any potential risks involved in their participation.

Competing Interests

Lewis J Macgregor, Malcolm M Fairweather, Ryan M Bennett and Angus M Hunter declare that they have no competing interests.

\section{Publisher's Note}

Springer Nature remains neutral with regard to jurisdictional claims in published maps and institutional affiliations.

\section{Author details \\ ${ }^{1}$ Faculty of Health Sciences and Sport, University of Stirling, Stirling, UK. ${ }^{2}$ SportScotland: Scottish Institute of Sport, Stirling, UK.}

Received: 12 December 2017 Accepted: 27 May 2018

Published online: 08 June 2018

\section{References}

1. Dommerholt J, Finnegan M, Grieve R, Hooks T. A critical overview of the current myofascial pain literature-January 2016. J Bodyw Mov Ther. 2016; 20(1):156-67.

2. Kim K, Park S, Goo B-O, Choi S-C. Effect of self-myofascial release on reduction of physical stress: a pilot study. J Phys Ther Sci. 2014;26(11):1779-81. 
3. Rodríguez-Fuentes I, De Toro FJ, Rodríguez-Fuentes G, de Oliveira IM, Meijide-Failde R, Fuentes-Boquete IM. Myofascial release therapy in the treatment of occupational mechanical neck pain: a randomized parallel group study. Am J Phys Med Rehabil. 2016;95(7):507-15.

4. Beardsley C, Škarabot J. Effects of self-myofascial release: a systematic review. J Bodyw Mov Ther. 2015;19(4):747-58.

5. Cheatham SW, Kolber MJ, Cain M, Lee M. The effects of self-myofascial release using a foam roll or roller massager on joint range of motion, muscle recovery, and performance: a systematic review. Int J Sports Phys Ther. 2015;10(6):827.

6. Healey KC, Hatfield DL, Blanpied P, Dorfman LR, Riebe D. The effects of myofascial release with foam rolling on performance. J Strength Cond Res. 2014;28(1):61-8.

7. Jay K, Sundstrup E, Søndergaard SD, Behm D, Brandt M, Særvoll CA, et al. Specific and cross over effects of massage for muscle soreness: randomized controlled trial. Int J Sports Phys Ther. 2014;9(1):82-91.

8. Pearcey GE, Bradbury-Squires DJ, Kawamoto J-E, Drinkwater EJ, Behm DG, Button DC. Foam rolling for delayed-onset muscle soreness and recovery of dynamic performance measures. J Athl Train. 2015:50(1):5-13.

9. MacDonald GZ, Button DC, Drinkwater EJ, Behm DG. Foam rolling as a recovery tool after an intense bout of physical activity. Med Sci Sports Exerc. 2014:46(1):131-42.

10. Bradbury-Squires DJ, Noftall JC, Sullivan KM, Behm DG, Power KE, Button DC. Roller-massager application to the quadriceps and knee-joint range of motion and neuromuscular efficiency during a lunge. J Athl Train. 2015; 50(2):133-40.

11. Sullivan K, Silvey D, Button DC, Behm D. Roller-massager application to the hamstrings increases sit-and-reach range of motion within five to ten seconds without performance impairments. International Journal of Sports Physical Therapy. 2013;8(3):228-36.

12. Janot J, Malin B, Jordan M, Cook R, Draeger A, Hagenbucher J, et al. Effects of self myofascial release \& static stretching on anaerobic power output. J Fit Res. 2013;2(1):2

13. Peacock CA, Krein DD, Silver TA, Sanders GJ, von Carlowitz K-PA. An acute bout of self-myofascial release in the form of foam rolling improves performance testing. International Journal of Exercise Science. 2014;7(3):202.

14. Roylance DS, George JD, Hammer AM, Rencher N, Fellingham GW, Hager $\mathrm{RL}$, et al. Evaluating acute changes in joint range-of-motion using selfmyofascial release, postural alignment exercises, and static stretches. International Journal of Exercise Science. 2013;6(4):6.

15. Škarabot J, Beardsley C, Štirn I. Comparing the effects of self-myofascial release with static stretching on ankle range-of-motion in adolescent athletes. International Journal of Sports Physical Therapy. 2015;10(2):203.

16. Couture G, Karlik D, Glass SC, Hatzel BM. The effect of foam rolling duration on hamstring range of motion. Open Orthop J. 2015:9:450-5.

17. Behm DG, Peach A, Maddigan M, Aboodarda SJ, DiSanto MC, Button $D C$, et al. Massage and stretching reduce spinal reflex excitability without affecting twitch contractile properties. J Electromyogr Kinesiol 2013;23(5):1215-21.

18. Hunter A, Watt JM, Watt V, Galloway S. Effect of lower limb massage on electromyography and force production of the knee extensors. Br J Sports Med. 2006;40(2):114-8.

19. Ditroilo M, Hunter AM, Haslam S, De Vito G. The effectiveness of two nove techniques in establishing the mechanical and contractile responses of biceps femoris. Physiol Meas. 2011;32(8):1315.

20. Pišot R, Narici MV, Šimunič B, De Boer M, Seynnes O, Jurdana M, et al. Whole muscle contractile parameters and thickness loss during 35-day bed rest. Eur J Appl Physiol. 2008;104(2):409-14.

21. Murray AM, Jones TW, Horobeanu C, Turner AP, Sproule J. Sixty seconds of foam rolling does not affect functional flexibility or change muscle temperature in adolescent athletes. International Journal of Sports Physical Therapy. 2016;11(5):765.

22. Martínez-Cabrera FI, Núñez-Sánchez FJ. Acute effect of a foam roller on the mechanical properties of the rectus femoris based on tensiomyography in soccer players. International Journal of Human Movement and Sports Sciences. 2016:4(2):26-32.

23. Cavanaugh MT, Döweling A, Young JD, Quigley PJ, Hodgson DD, Whitten JH, et al. An acute session of roller massage prolongs voluntary torque development and diminishes evoked pain. Eur J Appl Physiol. 2017;117(1):109-17.

24. Cheatham SW, Kolber MJ. Does self-myofascial release with a foam rol change pressure pain threshold of the ipsilateral lower extremity antagonist and contralateral muscle groups? An Exploratory Study. J Sport Rehabil. 2018;27(2):165-69.

25. Freiwald J, Baumgart C, Kühnemann M, Hoppe MW. Foam-rolling in sport and therapy-potential benefits and risks: part 1-definitions, anatomy, physiology, and biomechanics. Sports Orthopaedics and Traumatology. 2016;32(3):258-66

26. Macgregor LJ, Ditroilo M, Smith IJ, Fairweather MM, Hunter AM. Reduced radial displacement of the gastrocnemius medialis muscle after electrically elicited fatigue. J Sport Rehabil. 2016;25(3):241-7.

27. Tous-Fajardo J, Moras G, Rodríguez-Jiménez S, Usach R, Doutres DM, Maffiuletti NA. Inter-rater reliability of muscle contractile property measurements using non-invasive tensiomyography. J Electromyogr Kinesiol. 2010;20(4):761-6.

28. Šimunič B. Between-day reliability of a method for non-invasive estimation of muscle composition. J Electromyogr Kinesiol. 2012:22(4):527-30.

29. Gleeson N, Mercer T. The utility of isokinetic dynamometry in the assessment of human muscle function. Sports Med. 1996:21(1):18-34

30. Hermens HJ, Freriks B, Disselhorst-Klug C, Rau G. Development of recommendations for SEMG sensors and sensor placement procedures. J Electromyogr Kinesiol. 2000;10(5):361-74.

31. Balshaw TG, Hunter AM. Evaluation of electromyography normalisation methods for the back squat. J Electromyogr Kinesiol. 2012;22(2):308-19.

32. Hunter AM, Galloway SD, Smith IJ, Tallent J, Ditroilo M, Fairweather MM, et al. Assessment of eccentric exercise-induced muscle damage of the elbow flexors by tensiomyography. J Electromyogr Kinesiol. 2012;22(3):334-41.

33. Knapik JJ, Mawdsley RH, Ramos MU. Angular specificity and test mode specificity of isometric and isokinetic strength training. J Orthop Sports Phys Ther. 1983;5(2):58-65.

34. Halperin I, Pyne DB, Martin DT. Threats to internal validity in exercise science: a review of overlooked confounding variables. Int J Sports Physiol Perform. 2015;10(7):823-9.

35. Curran PF, Fiore RD, Crisco JJ. A comparison of the pressure exerted on soft tissue by 2 myofascial rollers. J Sport Rehabil. 2008;17(4):432-42.

36. Chaouachi A, Padulo J, Kasmi S, Othmen AB, Chatra M, Behm DG. Unilateral static and dynamic hamstrings stretching increases contralateral hip flexion range of motion. Clin Physiol Funct Imaging. 2017:37(1):23-29.

37. Dideriksen JL, Enoka RM, Farina D. Neuromuscular adjustments that constrain submaximal EMG amplitude at task failure of sustained isometric contractions. J Appl Physiol. 2011;111(2):485-94.

38. Moritani T, Muro M, Nagata A. Intramuscular and surface electromyogram changes during muscle fatigue. J Appl Physiol. 1986;60(4):1179-85.

39. Arabadzhiev TI, Dimitrov VG, Dimitrova NA, Dimitrov GV. Interpretation of EMG integral or RMS and estimates of "neuromuscular efficiency" can be misleading in fatiguing contraction. J Electromyogr Kinesiol. 2010;20(2):223-32.

40. Adam A, De Luca CJ. Firing rates of motor units in human vastus lateralis muscle during fatiguing isometric contractions. J Appl Physiol. 2005;99(1):268-80.

41. Dias da Silva S, Gonçalves M. Dynamic and isometric protocols of knee extension: effect of fatigue on the EMG signal. Electromyogr Clin Neurophysiol. 2005:46(1):35-42

42. Trajano GS, Seitz L, Nosaka K, Blazevich AJ. Contribution of central vs. peripheral factors to the force loss induced by passive stretch of the human plantar flexors. J Appl Physiol (1985). 2013;115(2):212-8.

43. Vigotsky AD, Halperin I, Lehman GJ, Trajano GS, Vieira TM. Interpreting signal amplitudes in surface electromyography studies in sport and rehabilitation sciences. Front Physiol. 2017:8:985.

44. Feland J, Marin H. Effect of submaximal contraction intensity in contractrelax proprioceptive neuromuscular facilitation stretching. Br J Sports Med. 2004;38(4):e18-e.

45. Sharman MJ, Cresswell AG, Riek S. Proprioceptive neuromuscular facilitation stretching : mechanisms and clinical implications. Sports Med. 2006;36(11):929-39.

46. Ranatunga K, Sharpe B, Turnbull B. Contractions of a human skeletal muscle at different temperatures. J Physiol. 1987;390:383.

47. Knight CA, Rutledge CR, Cox ME, Acosta M, Hall SJ. Effect of superficial heat, deep heat, and active exercise warm-up on the extensibility of the plantar flexors. Phys Ther. 2001:81(6):1206.

48. Morales-Artacho AJ, Lacourpaille L, Guilhem G. Effects of warm-up on hamstring muscles stiffness: cycling vs foam rolling. Scand J Med Sci Sports. 2017;27(12):1959-69.

49. Halperin I, Aboodarda SJ, Button DC, Andersen LL, Behm DG. Roller massager improves range of motion of plantar flexor muscles without 
subsequent decreases in force parameters. Int I Sports Physiol Ther. 2014; 9(1):92-102.

50. Bushell JE, Dawson SM, Webster MM. Clinical relevance of foam rolling on hip extension angle in a functional lunge position. The Journal of Strength \& Conditioning Research. 2015;29(9):2397-403.

51. Mohr AR, Long BC, Goad CL. Effect of foam rolling and static stretching on passive hip-flexion range of motion. J Sport Rehabil. 2014;23(4):296-9.

\section{Submit your manuscript to a SpringerOpen ${ }^{\circ}$} journal and benefit from:

- Convenient online submission

- Rigorous peer review

- Open access: articles freely available online

- High visibility within the field

- Retaining the copyright to your article

Submit your next manuscript at $\gg$ springeropen.com 